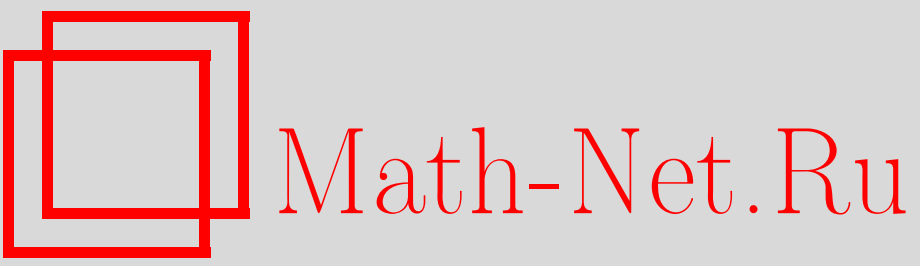

И. В. Артельных, Произведения Масси и спектральная последовательность Бухштабера, УМН, 2000, том 55, выпуск 3, 165-166

DOI: https://doi.org/10.4213/rm294

Использование Общероссийского математического портала Math-Net.Ru подразумевает, что вы прочитали и согласны с пользовательским соглашением

http://www.mathnet.ru/rus/agreement

Параметры загрузки:

IP: 54.162 .85 .209

26 апреля 2023 г., 16:18:34 


\title{
ПРОИЗВЕДЕНИЯ МАССИ И СПЕКТРАЛЬНАЯ ПОСЛЕДОВАТЕЛЬНОСТЬ БУХШТАБЕРА
}

\author{
И.В. АРТЕЛЬНЫх
}

В 1978 г. в [1] была установлена связь между алгеброй Ландвебера-Новикова $S$ и алгеброй Ли $L_{1}\left(W_{1}\right)$ формальных векторных полей на прямой, а именно, доказан изоморфизм: $S^{2 q} \otimes \mathbb{Q} \cong$ $U\left(L_{1}\right)^{q}$, где $U\left(L_{1}\right)$ - универсальная обертьвающая алгебра алгебры Ли $L_{1}\left(W_{1}\right)$, и, следовательно, $\operatorname{Ext}_{S}^{*}(\mathbb{Z}, \mathbb{Z}) \otimes \mathbb{Q}=H^{*}\left(L_{1}, \mathbb{Q}\right)$. Когомологии алгебры Ли $L_{1}\left(W_{1}\right)$ были вычислены в [2]: $H^{n, k}\left(L_{1}, \mathbb{Q}\right)=\mathbb{Q}$ для $k=\frac{3 n^{2} \pm n}{2}$ и $H^{n, k}\left(L_{1}, \mathbb{Q}\right)=0$ для $k \neq \frac{3 n^{2} \pm n}{2}$. По размерностным соображениям умножение в кольце $H^{*, *}(S)=\operatorname{Ext}_{S}^{*, *}(\mathbb{Z}, \mathbb{Z}) \otimes \mathbb{Q}$ тривиально. Обозначим через $x_{+}^{n}$ и $x_{-}^{n}$ старшую и младшую по второй градуировке образуюшие в группе $H^{n}(S \otimes \mathbb{Q})$. В начале 70-х годов В. М. Бухштабером была высказана гипотеза о том, что образующие группы $H^{n}(S)$ представляют собой произведения Масси от образующих группы $H^{1}(S)$. Напомним определение произведения Масси [3]. Пусть $c_{i} \in H^{n_{i}}(S), i=1, \ldots, r, n_{i}>0$, и существуют коцепи $c_{i, j}$, $i=1, \ldots, r-1, j=1, \ldots, r-(i-1)$ такие, что $c_{1, j} \in c_{j}$ и

$$
d c_{i, j}=\sum_{l=1}^{i-1} \bar{c}_{l, j} c_{i-l, j+l}
$$

где $\bar{c}_{i, j}=(-1)^{\operatorname{deg} c_{i, j}+1} c_{i, j}$. Множество $\left\{c_{i, j}\right\}$ называется определяюшей системой для произведения Масси. В этом случае $\sum_{l=1}^{r-1} \bar{c}_{l, 1} c_{r-l, l+1}$ является коциклом и определяет когомологический класс $y \in H^{n_{1}+\cdots+n_{r}-(r-2)}$, назьваемый реализацией произведения Масси $\left\langle c_{1}, \ldots, c_{r}\right\rangle$. Как видно из определения, присутствует некий произвол при выборе элементов $c_{i, j}$. Этот произвол приводит к тому, что произведение Масси определено неоднозначно. Таким образом, гипотеза В.М. Бухштабера может быть разбита на две проблемы. Первая: найти представления образующих групп $H^{n}(S)$ в виде реализаций произведений Масси. Вторая: найти среди таких произведений те, которые не допускают нулевых реализаций.

Решение первой проблемы было получено в [4], где доказано, что

$$
x_{-}^{n} \in \mathbb{Q}\langle x_{+}^{n-1}, \underbrace{x_{-}^{1}, \ldots, x_{-}^{1}}_{2 n-1}\rangle, \quad x_{+}^{n} \in \mathbb{Q}\langle x_{+}^{n-1}, \underbrace{x_{-}^{1}, \ldots, x_{-}^{1}}_{3 n-1}\rangle
$$

для всех $n \geqslant 2$. Вторая проблема в статье [4] не рассматривалась, и полученные в ней результаты не дают решения этой проблемы, так как произведение $\langle x_{+}^{n-1}, \underbrace{x_{-}^{1}, \ldots, x_{-}^{1}}_{2 n-1}\rangle$ допускает реализации, равные нулю, т.е. возможно построить такую определяющую систему, что результирующий коцикл будет когомологичен нулю.

Таким образом, оставалась проблема описания образуюших кольца $H^{*}(S)$ в терминах произведений Масси, не допускающих нулевых реализаций. Следующий резултат дает решение этой проблемы для $x_{-}^{n}, n \geqslant 2$, и $x_{+}^{2 l+1}, l \geqslant 1$.

Теорема 1. Имеют место следующие произведения Масси, не допускающие нулевых

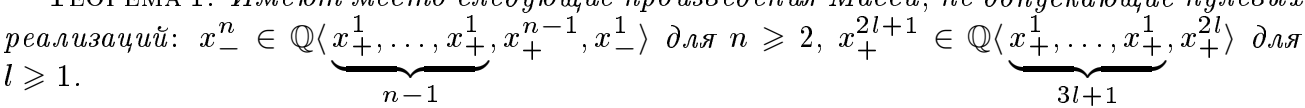

В [4] без доказательства упоминается, что образующие $x_{+}^{2 l+1}$ имеют представления, указанные в теореме 1 , но вопрос о несуществовании нулевых реализаций не затрагивается.

Доказательство теоремы 1 основано на рассмотрении взаимосвязи произведений Масси с дифоференциалами спектральной последовательности Бухштабера. Рассмотрим алгебру Ландвебера-Новикова $S$ и ее действие на колце кобордизмов $\Omega_{U}$. Следуя [5], строим в кольце $\Omega_{U} \otimes \mathbb{Q}$ фильтрацию $N_{0} \subset N_{1} \subset \cdots$, где $N_{k}=\left\{y \in \Omega_{U} \otimes \mathbb{Q} \mid s_{\omega}(y) \in N_{k-1}, \forall s_{\omega} \in S\right\}, \mathrm{c}$ начальным членом $N_{0}=\mathbb{Z}$. Предел этой фильтрации есть подкольцо $\Omega_{U}(\mathbb{Z}) \subset \Omega_{U} \otimes \mathbb{Q}$, причем $\Omega_{U}(\mathbb{Z}) \cong \mathbb{Z}\left[\beta_{1}, \beta_{2}, \ldots\right]$, где $\beta_{1}, \beta_{2}, \ldots$ - коэффициенты характера Чженя-Дольда согласно 
[1], [5]. В итоге получаем триградуированную спектральную последовательность для когомологий алгебры $S$, ассоциированную с этой фильтрацией. Третья градуировка наследуется из второй градуировки коцепного комплекса алгебры $S$ с коэффициентами $\Omega_{U}(\mathbb{Z})$. Такая спектральная последовательность называется спектральной последовательностью Бухштабера (СПБ) [6]. Для любого $S$-подмодуля $V \subset \Omega_{U}(\mathbb{Z})$ такого, что $\mathbb{Z}=N_{0} \subset V$, имеют место индуцированная фильтрация и соответствующая СПБ, сходящаяся к $H^{*}(S ; V)$. Наше доказательство опирается на так называемые ниточные представления алгебры Ландвебера-Новикова $S$ из [4]. Когомологии алгебры $S$ с коэффициентами в этих представлениях будем обозначать далее через $H^{*}(S ; V)$. В рассматриваемом случае определяющая система для произведения Масси строится без препятствий до тех пор, пока $\operatorname{deg} c_{i, j}<3 n^{2}-n$, так как $H^{n, q}(S)=0$ для $q<3 n^{2}-n$. Первое препятствие появляется при $\operatorname{deg} c_{i, j}=3 n^{2}-n$. В этом случае согласно предложению 2.1 из [4] уравнение (1) разрешимо тогда и только тогда, когда дифференциал $E_{2 n-1}^{2 n-1,-3 n+2,3 n^{2}-n} \rightarrow E_{2 n-1}^{0,-n, 3 n^{2}-n}$ в СПБ для $H^{*}(S ; V)$ равен нулю. Если он равен 0, т.е. уравнение (1) разрешимо, то определяющая система строится без препятствий, пока $\operatorname{deg} c_{i, j}<3 n^{2}+n$, так как $H^{n, q}(S)=0$ для $3 n^{2}-n<$ $q<3 n^{2}+n$. Второе препятствие появляется при $\operatorname{deg} c_{i, j}=3 n^{2}+n$. В этом случае уравнение (1) опять разрешимо тогда и только тогда, когда дифференциал $E_{3 n-1}^{3 n-1,-4 n+2,3 n^{2}+n} \rightarrow$ $E_{3 n-1}^{0,-n, 3 n^{2}+n}$ в СПБ для $H^{*}(S ; V)$ равен нулю. В итоге вопрос о существовании произведений Масси, выписанных в теореме 1 , сводится к вопросу о существовании некоторых элементов в $H^{*}(S ; V)$. В [4] выписаны минимальная свободная резольвента и явные формулы для дифференциалов, позволяющие вычислить $H^{*}(S ; V)$. Так доказывается существование приведенных в формулировке теоремы 1 произведений Масси. Доказательство того, что эти произведения Масси не допускают нулевых реализаций, основано на рассмотрении второй градуировки в когомологиях алгебры $S$. Причем существенную роль в этом доказательстве играет особый подбор элементов приведенных произведений Масси. Например, для произведений Масси, указанных в [4], подобное доказательство уже не проходит.

Для рассматриваемых представлений удалось в явном виде описать соответствующие им $S$-подмодули $V \subset \Omega_{U}(\mathbb{Z})$. Это дало возможность вычислить некоторые дифференциалы в СПБ для $H^{*}\left(S ; \Omega_{U}(\mathbb{Z})\right)$.

TЕорема 2. $d_{n}^{n,-2 n+1}\left(y_{1} y_{2}^{n-1} \otimes x_{+}^{n-1}\right)=x_{-}^{n}$ для $n \geqslant 2, d_{3 l+1}^{3 l+1,-5 l-1}\left(y_{2}^{3 l+1} \otimes x_{+}^{2 l}\right)=$ $x_{+}^{2 l+1}$ для $l \geqslant 1$. Здесь $y_{1}=\beta_{1}, y_{2}=\beta_{2}-\beta_{1}^{2}$.

Методы, развитые для доказательства теоремы 1 , позволили получить результаты о произведениях Масси в когомологиях замечательных нильмногообразий [7]. В [8] эти многообразия описаны в виде последовательности расслоений $M^{k+1} \rightarrow M^{k}, \operatorname{dim} M^{k}=k$, таких, что $H^{*}\left(\stackrel{\lim }{\longleftarrow} M^{k}\right)$ $=H^{*}(S)$. В $H^{2}\left(M^{k}\right)$ существует нетривиальньй элемент $\Omega_{k}$, определяемьй формой кривизны связности расслоения $M^{k+1} \rightarrow M^{k}$, который задает для $k=2 m$ симплектическую структуру на $M^{k}$.

Теорема 3. В $H^{*}\left(M^{k}\right)$ имеют место следующие произведения Масси, не допускающие нулевьх реализаций $\Omega_{k} \in \mathbb{Q}\langle\underbrace{x_{-}^{1}, \ldots, x_{-}^{1}}_{k-1}, x_{+}^{1}\rangle$.

\section{СПИСОК ЛИТЕРАТУРЫ}

[1] Бухштабер В. М., Шокуров А. В. // Функц. анализ и его прил. 1978. Т. 12. № 3. С. 1-11. [2] Гончарова Л. В. // Функц. анализ и его прил. 1973. Т. 7. № 2. С. 6-14. [3] Kraines D. // Trans. Amer. Math. Soc. 1966. V. 124. P. 431-449. [4] Feigin B. L., Fuchs D. B., Retakh V.S. // Lecture Notes in Math. 1988. V. 1346. P. 13-31. [5] Бухштабер В. M. // Матем. сб. 1970. Т. 87. С. 575-595. [6] Фукс Д. Б. Когомологии бесконечномерных алгебр Ли. M.: Наука, 1984. [7] Babenko I. K., Taimanov I. A. On nonformal simply connected symplectic manifolds // SFB 288 Preprint 358. Berlin, 1998. [8] Бухштабер В. М. // УМН. 1999. T. 54. № 4. C. 161-162. 